\title{
Uma análise das motivações dos voluntários em Associações Humanitárias de Bombeiros
}

\section{An analysis of the motivations of volunteers at Humanitarian Firefighters Associations}

Artigo Original | Original Article

Vasco Almeida, PhD (1a), Fernanda Daniel, PhD (2b), Henrique Fernandes (3c)

(1) Instituto Superior Miguel Torga; Centro de Estudos Sociais da Universidade de Coimbra.

(2) Instituto Superior Miguel Torga; Centro de Estudos e Investigação em Saúde da Universidade de Coimbra.

(3) Instituto Superior Miguel Torga.

(a) Revisão da Literatura, Discussão dos Resultados/Conclusão e Redação.

(b) Análise dos dados, Redação e Sugestões/Recomendações Gerais

(c) Revisão da Literatura, Redação e Revisão geral do texto

Autor para correspondência | Corresponding author: Vasco Almeida - vascoalmeida@ismt.pt

\section{Palavras-Chave}

Fatores de motivação Bombeiros Voluntários Portugal

\section{Keywords}

Motivation factors Volunteer firefighters Portugal

\section{RESUMO}

Objetivos: Identificar os fatores que motivam bombeiros para o voluntariado e perceber de que forma características tais como sexo, idade, grau de escolarização, rendimento e anos de voluntariado, podem influenciar os tipos de motivação.

Metodologia: Participaram 126 bombeiros voluntários de duas Associações Humanitárias, com uma idade média de $36,06$ anos ( $D P=12,46)$, a maioria dos participantes pertence ao sexo masculino $(n=84 ; 66,7 \%)$, é casada ( $n=53$; $42,1 \%)$, tem como habilitações o ensino secundário ( $n=74 ; 58,7 \%), 83(65,9 \%)$, aufere um rendimento mensal compreendido entre os $500 €$ e os $1000 €$ e encontra-se há mais de dez anos na respetiva Associação como voluntário/a ( $n=66 ; 52,4 \%)$. Os instrumentos utilizados foram um questionário de caracterização sociodemográfica e uma escala composta por 17 afirmações elaborados por Anne Ward e Donal Mckillop (2011).

Resultados: A escala apresenta boas características psicométricas $(\alpha=0,83)$. É nos "resultados" que obtemos os valores médios mais elevados e mais baixos - indicador "Altruísta - K" $(M=6,27$; DP =1,10) versus "Necessidades - Q" $(M=4,23 ; D P=2,39)$. Na análise das variáveis motivacionais, segundo as características sociodemográficas, verificamos que os indicadores que apresentam maior número de diferenças, estatisticamente significativas, são o "Egoísta" do "Capital humano" (H), o "Altruísta" (K, L, M) e a "Necessidade" (O) dos "Resultados".

Discussão e Conclusões: Os resultados globais do nosso estudo mostram que o altruísmo é o fator dominante na explicação do comportamento dos bombeiros voluntários, o que é consistente com investigações anteriores sobre a motivação para o voluntariado. Todavia, quando desagregamos a análise através das características dos voluntários, os resultados nem sempre coincidem com aqueles que se encontram na literatura. $O$ contexto sociocultural e as especificidades da própria atividade dos bombeiros voluntários podem ser responsáveis pelas diferenças encontradas. Serão precisos mais estudos comparativos a nível internacional e que tenham em conta as características específicas da atividade dos bombeiros voluntários, nomeadamente a questão do risco.

\section{ABSTRACT}

Objectives: To identify the factors that motivate firefighters to volunteer in Portugal and to understand how their characteristics, such as their gender, age, education level, income and years of volunteering, can influence the types of motivation.

Methodology: A total of 126 volunteer firefighters from two Humanitarian Associations participated, with a mean age of 36.06 years $(S D=12.46)$, the majority of participants is male $(n=84,66.7 \%)$, is married $(n=53,42.1 \%)$, has secondary education $(n=74,58.7 \%), 83(65.9 \%)$ receive a monthly income between $€ 500$ and $€ 1000$ and has been in the Association for more than 10 years as volunteers ( $n=66,52.4 \%$ ). The instruments used were a sociodemographic characterization questionnaire and a scale composed of 17 statements elaborated by Anne Ward and Donal Mckillop (2011).

Results: The scale has good psychometric characteristics $(\alpha=0.83)$. In the "results" we obtain the highest and lowest mean values - "Altruistic - K" indicator $(M=6.27, S D=1.10)$ versus "Needs - Q" $(M=4.23, S D=2.39)$. In the analysis of motivational variables, according to the sociodemographic characteristics, the indicators with the highest number of statistically significant differences are the "Selfish" of "Human capital" (H), the "Altruistic" (K, L, M) and the "Need" (O) of "Results".

Discussion and Conclusions: The altruism is the dominant factor in explaining the behavior of volunteer firefighters, consistent with previous research on motivation for volunteering. However, when we disaggregate the analysis the results do not always coincide with those found in the literature. The sociocultural context and the specificities of the volunteers' own activity may be responsible for the differences found. Further comparative studies at international level are necessary, and taking into account the specific characteristics of voluntary firefighter's activity, namely the issue of risk. 


\section{INTRODUÇÃO}

Em 2015, estavam referenciados em Portugal 472 corpos de bombeiros e um total de 28957 bombeiros. O Continente apresentava 445 corpos de bombeiros (27 427 bombeiros), a Região Autónoma dos Açores 17 (886 bombeiros) e a Região Autónoma da Madeira 10 (644 bombeiros). Refira-se que a maioria dos bombeiros são voluntários, representando $70,7 \%$ do total nacional (INE, 2017).

Uma parte muito significativa das organizações não lucrativas, como é o caso das Associações Humanitárias de Bombeiros, debate-se com as questões de recrutamento e retenção dos voluntários (Ward e McKillop, 2010, 2011; Jager, Kreutzer e Beyes, 2009). Em Portugal, um país que tem sido fustigado todos os anos por um número elevado de incêndios, alguns de consequências trágicas, o recrutamento e retenção de bombeiros voluntários reveste-se de uma particular relevância. Vários autores têm chamado a atenção para o facto de que o estudo do perfil e das motivações dos voluntários poder melhorar significativamente as estratégias do seu recrutamento e retenção. Por exemplo, o estudo de Anderson (2003) sobre o voluntariado dos estudantes em hospitais mostra que a ligação que os indivíduos sentem com a organização determina a opção pela continuação do voluntariado. De forma idêntica, Tidwell (2005), utilizando como suporte teórico a teoria da identidade social, chega à conclusão que a retenção de voluntários está associada ao seu vínculo com a organização.

Normalmente, distinguem-se vários tipos de motivação, muitas vezes associados a modelos microeconómicos de investimento e de consumo (ver, por exemplo, Ziemek, 2006). A motivação, segundo o modelo de investimento, explica a oferta de voluntariado pelo desejo que os indivíduos têm de melhorar o seu capital humano, seja para ampliarem as possibilidades de emprego, seja para obterem uma mais-valia salarial. 0 modelo de consumo, por outro lado, enfatiza a utilidade subjetiva que é retirada pelo prazer de dar e ajudar os outros. É também comum, na literatura especializada, relacionar os diferentes tipos ou modelos de motivação com diversas características dos voluntários. De uma forma geral, alguns atributos dos voluntários, designadamente o sexo, a idade, o grau de escolarização, o rendimento e os anos de voluntariado, têm sido relacionados com diferentes tipos de motivação.

Em relação às diferenças segundo o sexo, são vários os estudos que apontam que as mulheres, em relação aos homens, são mais motivadas para o voluntariado por razões altruístas (Eckel e Grossman, 2008; Themudo, 2006; Wilson, 2000). Por outro lado, as principais motivações para o voluntariado nos homens residem nas razões sociais (Maclaran e Caterrall, 2000) e no interesse próprio
(Ziemek, 2006). Porém, os resultados são, por vezes, bastante ambíguos. Por exemplo, Andreoni e Vesterlund (2001) defendem que a questão sobre quem é "o sexo mais generoso" não tem uma resposta fácil. Os resultados investigação destes autores, baseados na teoria dos jogos, salientam que o altruísmo depende do tipo de voluntariado. Quando o altruísmo envolve custos mais elevados, as mulheres são mais generosas, mas quando implica custos mais baixos os homens tendem a ser mais altruístas. Para outros autores, a cultura institucional e o papel das mulheres na sociedade são elementos determinantes na configuração das preferências e das tendências altruístas (Gneezy, Leonard e List, 2009; Gong, Yan e Yang, 2014). Assim, o padrão frequentemente encontrado nos estudos norte-americanos caracterizado por um maior altruísmo das mulheres, não pode ser universalizado para todo o tipo de sociedades. $\mathrm{Na}$ verdade, os autores mostram que, em determinadas sociedades de características matriarcais, as mulheres parecem ser mais guiadas pelo interesse próprio e menos pelo altruísmo.

É também avançado por vários autores que a idade é um fator diferenciador no tipo de motivação para o voluntariado. Ziemek (2006) e Peterson (2004) mostram que os jovens tendem a apresentar motivações mais ligadas a um modelo de investimento pessoal, isto é, veem no voluntariado uma forma de elevar o seu capital humano e as suas perspetivas de empregabilidade. De forma idêntica, Nichols e Raltson (2016) concluem, num estudo sobre o voluntariado nos Jogos Olímpicos de 2012, em Londres, que os voluntários de escalões etários mais elevados tendem a expressar valores altruístas, enquanto as camadas etárias mais jovens são maioritariamente motivadas pela perspetiva de melhorar a sua empregabilidade. Todavia, esta dicotomia torna-se mais esbatida quando os autores combinam a análise quantitativa com métodos qualitativos, demonstrando que os voluntários de maior idade podem também ser motivados pelo interesse próprio. Okun e Schuktz (2003), mostram que a relação entre a idade, os valores e as razões sociais que motivam voluntariado está longe de ser linear, o que contraria uma boa parte dos estudos sobre o voluntariado.

O nível de escolarização também é uma das variáveis apontadas como influenciadora do tipo de motivação que explicam as atividades de voluntariado. As hipóteses adiantadas na literatura sustentam que os fatores determinantes para o voluntariado nos grupos sociais com níveis mais baixos de escolarização estão ligados à vontade de ganhar experiência educacional e prática, elevando dessa forma o seu perfil na comunidade (Clary, Snyder e Stukas, 1996). Por sua vez, os indivíduos com níveis de escolarização mais elevados estão dominantemente 
motivados por razões altruístas (Ward e Mckilop, 2011; Wilson, 2000).

Alguns estudos sobre a relação entre o nível de rendimento e a motivação para o voluntariado têm mostrado que existe uma correlação positiva entre aquelas duas variáveis. Desta forma, indivíduos que auferem rendimentos mais elevados encontram-se, globalmente, mais motivados para dedicarem algum do seu tempo às atividades de voluntariado do que aqueles que se situam nos escalões de rendimento mais baixo (Dettoleneare, Baert e Willems, 2017). Porém, escasseiam as investigações sobre a relação entre os diferentes tipos de motivação para o voluntariado e os níveis de rendimento. As referências que existem sobre esta relação apontam no sentido de que os escalões mais baixos de rendimento tendem a voluntariar-se para elevar o seu nível de capital humano e, assim, aumentarem as possibilidades de obtenção de uma mais valia salarial, enquanto as razões que explicam as atividades de voluntariado das classes de rendimentos mais elevado estão, sobretudo, ligadas a razões altruístas e às necessidades de interação social. No entanto, uma vez mais os resultados empíricos são, por vezes, ambíguos e aquelas relações nem sempre são lineares (Hackl, Halla e Pruckner, 2007).

A relação entre o tempo de permanência na organização e as determinantes do voluntariado é, igualmente, um dos temas abordados na literatura sobre o voluntariado. Os resultados encontrados em diferentes estudos apontam para a distinção entre dois tipos de voluntários: os iniciantes e os experientes. É sugerido que os motivos que justificam a oferta de trabalho voluntário evoluem ao longo do tempo (Thomas e Finch, 1990; Tschirhart, Mesh, Miller e Lee, 2001), defendendo-se que os voluntários de longo prazo são mais motivados pelo altruísmo, enquanto os voluntários que estão há menos tempo na organização são mais estimulados pelas possibilidades de elevar o seu nível de capital humano e pelo desejo de desenvolverem as suas interações sociais (Ward e Mckilop, 2011).

O nosso estudo centra-se neste debate e tem como objetivo perceber, em primeiro lugar, quais os fatores que, de uma forma geral, motivam os bombeiros para o voluntariado e, em segundo lugar, perceber de que forma as características dos voluntários, nomeadamente no que dizem respeito ao sexo, idade, grau de escolarização, rendimento e anos de voluntariado, podem estar relacionadas com os diferentes tipos de motivação.

Como a motivação é um constructo complexo e difícil de entender na sua totalidade, seguimos no nosso estudo a proposta apresentada por Ward e Mckilop (2011). Estes autores utilizam um paradigma simplificado que utiliza um número relativamente reduzido de fatores chave na motivação, identificados na literatura, assumindo-se que explicam uma larga proporção das atividades de voluntariado. São assim identificados 17 fatores de motivação, mais à frente descritos (v. tabela 1), agrupados em três tipos de motivação: a motivação pelo ato do voluntariado, a motivação pelos resultados do voluntariado (onde se inclui o altruísmo) e a motivação para melhorar o capital humano.

\section{MATERIAL E MÉTODOS}

\section{Participantes}

Participaram na pesquisa os corpos ativos de duas associações de bombeiros voluntários $(n=126)$. Apresentam uma idade média de 36,06 anos ( $D P=12,46)$, a maioria dos participantes pertence ao sexo masculino ( $n=84 ; 66,7 \%)$, é casada $(n=53 ; 42,1 \%)$, tem como habilitações o ensino secundário ( $n=74 ; 58,7 \%), 83$ $(65,9 \%)$ aufere um rendimento mensal compreendido entre os $500 €$ e os $1000 €$ e encontra-se há mais de dez anos na associação respetiva como voluntários ( $n=66$; $52,4 \%$ ) (Tabela 1).

Tabela 1 Caracterização Sociodemográfica

\begin{tabular}{|c|c|c|}
\hline & \multicolumn{2}{|c|}{$M(D P)$} \\
\hline \multirow[t]{2}{*}{ Idade } & \multicolumn{2}{|c|}{$36,06(12,46)$} \\
\hline & $N \%$ & \\
\hline \multicolumn{3}{|l|}{ Sexo } \\
\hline Masculino & 84 & 66,7 \\
\hline Feminino & 41 & 32,5 \\
\hline Não respondeu & 1 & 0,8 \\
\hline \multicolumn{3}{|l|}{ Estado Civil } \\
\hline Solteiro(a) & 53 & 42,1 \\
\hline Casado(a)/união de facto & 55 & 43,7 \\
\hline Divorciado(a) & 15 & 11,9 \\
\hline Viúvo(a) & 3 & 2,4 \\
\hline \multicolumn{3}{|l|}{ Escolaridade } \\
\hline Ensino básico & 38 & 30,2 \\
\hline Ensino secundário & 74 & 58,7 \\
\hline Ensino superior $\left(1 .^{\circ}\right.$ e $2 .^{\circ}$ & 13 & 10,4 \\
\hline Não responde & 1 & 0,8 \\
\hline \multicolumn{3}{|l|}{ Rendimento Líquido Mensal } \\
\hline Entre $500-1000 €$ & 83 & 65,9 \\
\hline Entre $1000-1500 €$ & 15 & 11,9 \\
\hline Entre $1500-2500 €$ & 4 & 3,2 \\
\hline Mais de 2500 & 1 & 0,8 \\
\hline Não responde (inclui 1 & 23 & 18,3 \\
\hline \multicolumn{3}{|l|}{ Tempo na instituição } \\
\hline$<2$ anos & 12 & 9,5 \\
\hline 2-10 anos & 48 & 38,1 \\
\hline$>10$ anos & 66 & 52,4 \\
\hline
\end{tabular}

Nota. $n$ = Frequência; $M$ = Média; $D P$ = Desvio-padrão; \% = Percentagem. 


\section{Instrumento}

Os dados foram recolhidos através do questionário, elaborado por Anne Ward e Donal Mckillop (2011), que avalia a motivação para o voluntariado. Possui 17 afirmações pontuadas numa escala de Likert de 7 pontos cujo ponto mínimo é 1 (discordo totalmente) e o ponto máximo é 7 (concordo totalmente). O questionário encontra-se dividido em três tipos de motivação: "agir", "capital humano" e "resultados". A dimensão "agir" (A, B, C, D, E e F) apresenta três indicadores/áreas "prazer" ( $A, C$ e D), "interesse" (B) e "interação social" (E e F). O "capital humano" é medido através de três itens $(\mathrm{G}, \mathrm{H}$ e l) referente a dois indicadores/áreas "egoísta no plano material" (G e I) e "egoísta" (H). Por último, os "resultados" (J, K, L, M, N, O, P e Q) medido através de três indicadores/áreas "altruísta" (J, K, L e $M)$, "necessidade" ( $\mathrm{N}$ e O) e "dever moral" ( $\mathrm{P}$ e Q). No nosso estudo, o questionário obteve bons valores de consistência interna ( $\alpha /$ Total dos itens = 0,83; "Agir" = 0,74 ; “Capital Humano" = 0,60 e "Resultados" = 0,70).

\section{Análise estatística}

$\mathrm{Na}$ análise dos dados foi utilizado o Statistical Package for the Social Sciences (IBM-SPSS), versão 24. Calculámos medidas de estatísticas descritivas nomeadamente medidas de tendência central a par das medidas de dispersão. Os testes não paramétricos Kruskal-Wallis e o U de Mann-Whitney foram utilizados para determinar diferenças. Usou-se o nível de significância de 0,05.

Quadro 1

Tipos de Motivação no Voluntariado

\begin{tabular}{lll}
\hline \multicolumn{2}{l}{ Itens } & Tipos de motivação \\
\hline A. Sinto-me satisfeito a fazer voluntariado. & Agir (prazer) \\
B. Faço voluntariado porque é interessante. & Agir (interesse) \\
C. Fico feliz com as ações de voluntariado. & Agir (prazer) \\
D. Faço voluntariado porque gosto de estar ocupado. & Agir (prazer) \\
E. Faço voluntariado porque gosto de conhecer pessoas e da interação social. & Agir (social) \\
F. Voluntario-me porque conheço os outros voluntários. & Agir (social) \\
G. Ganho uma experiência educacional/cultural. & Capital humano (material-egoísta) \\
H. $\quad$ Ao voluntariar-me melhoro a minha imagem para com a comunidade. & Capital humano (egoísta) \\
I. Voluntario-me porque estou a ganhar experiência prática para um emprego & Capital humano (material-egoísta) \\
J. Estou a fazer algo útil. & Resultados/Saída (altruísta) \\
K. É uma grande oportunidade para ajudar quem mais precisa na comunidade. & Resultados/Saída (altruísta) \\
L. Estou a contribuir para uma sociedade melhor. & Resultados/Saída (altruísta) \\
M. Voluntario-me porque estou a contribuir para o futuro da empresa/ & Resultados/Saída (altruísta) \\
N. Faço voluntariado porque a empresa/organização teria dificuldade em & Resultados/Saída (necessidade) \\
O. Voluntario-me porque a empresa/organização beneficia com a minha & Resultados/Saída (necessidade) \\
P. $\quad$ Faco voluntariado porque já o fiz no passado ou a minha família o fez. & Resultados/Saída (dever moral) \\
Q. Faço voluntariado porque a minha família / amigos esperam isso de mim. & Resultados/Saída (dever moral) \\
\hline
\end{tabular}

Fonte: Ward e Mckillop (2011).

\section{RESULTADOS}

Como se pode observar, na Tabela 2, é nos "Resultados" que obtemos os valores médios simultaneamente mais elevados e mais baixos. $\mathrm{O}$ item $\mathrm{K}-$ "É uma grande oportunidade para ajudar quem mais precisa na comunidade", relativo ao indicador/área "altruísta", obtém a média superior $(M=6,27 ; D P=1,10)$ enquanto o item Q - "Faço voluntariado porque a minha família/amigos esperam isso de mim", indicador/área relativo ao "dever moral", obtém a média mais baixa $(M=4,23$; $D P=2,39)$.

A Tabela 3 apresenta as pontuações obtidas nos itens relativos à motivação para o voluntariado, segundo o sexo. É nos itens relativos ao "capital humano" e aos "resultados" que se observam diferenças estatisticamente significativas, quando comparamos os efetivos do sexo masculino com os do sexo feminino nos corpos de bombeiros. O teste Mann-Whitney revela diferenças estatisticamente significativas entre os elementos do sexo masculino $(M d=6 ; n=83)$ e do sexo feminino $(M d=5 ; n=$ 41) no item " $\mathrm{H}$ - Ao voluntariar-me melhoro a minha imagem para com a comunidade", indicador "egoísta" do "capital humano", $U=1280,00 ; z=-2,302 ; p=0,021$. São, igualmente, reportadas diferenças estatisticamente significativas, nos itens " $L$ - Estou a contribuir para uma sociedade melhor" e " $M$ - Voluntario-me porque estou a contribuir para o futuro da empresa/organização", indicadores "altruístas" dos "resultados" quando comparamos os elementos do sexo masculino $(M d=7 ; n=$ 
$83-\mathrm{L} ; M d=6, n=83-M)$ com os elementos do sexo feminino $(M d=6 ; n=41-L ; M d=5 ; n=41-M), U=1291,50$, $z=-2.376, p=0,018$ e $U=1138,50 ; z=-3,101, p=0,002$, respetivamente. No item "O - Voluntario-me porque a empresa/organização beneficia com a minha experiência", indicador "necessidade" dos "resultados" são igualmente detetadas diferenças estaticamente significativas entre os elementos do sexo masculino $(M d=6 ; n=83)$ e do sexo feminino $(M d=5 ; n=41) ; U=1253,00 ; z=-2,351 ; p=0,019$.

A Tabela 4 apresenta as pontuações obtidas nos itens relativos à motivação para o voluntariado segundo as idades, em classes. Nos itens "G - Ganho uma experiência cultural" - indicador "material-egoísta" do "capital humano" e "N - Faço voluntariado porque a empresa/ organização teria dificuldade em arranjar outros voluntários", indicador "necessidade" dos "resultados" obtemos diferenças estatisticamente significativas, quando comparamos as pontuações desses itens, consoante as classes etárias. O teste Kruskal-Wallis revela diferenças estatisticamente significativas nas pontuações do item " $\mathrm{G}$ Ganho uma experiência cultural" indicador "materialegoísta" do "capital humano", consoante as classes etárias (Gp1, $n=80:<40$ anos; Gp2, $n=40: 40-59$ anos; Gp3, $n=5$ : $60+$ anos $), \chi^{2}(2, n=125)=7,41, p=0,025$. As diferenças, no caso do item " $G$ - Ganho uma experiência educacional/cultural", são constatadas nas pontuações entre o Gp1 (< 40 anos) e o Gp2 (40-59 anos) ( $M d=6$ vs. $M d=7$ ), $U=1178,00 ; z=-2,477$; $p=0,013$. Já no item “ $N$ - Faço voluntariado porque a empresa/organização teria dificuldade em arranjar outros voluntários", indicador "necessidade" dos "resultados", verificamos diferenças estatisticamente significativas, $\chi_{2}^{2}(2, n$ $=125)=6,68, p=0,035$. As diferenças, estaticamente significativas, são obtidas quando comparamos as pontuações dos bombeiros com idades mais baixas Gp1 $(<$ 40 anos) e os bombeiros com idades mais elevadas Gp3 $(60+$ anos) $(M d=5$ vs. $M d=6), U=82,00 ; z=-2,240 ; p=$ 0,025 .

Tabela 2

Medidas de Tendência Central e de Dispersão dos Itens da Escala

\begin{tabular}{|c|c|c|c|c|c|c|c|c|c|c|c|c|c|c|c|c|c|}
\hline \multirow[b]{3}{*}{ Ordem } & \multicolumn{6}{|c|}{ Agir } & \multicolumn{3}{|c|}{ Capital Humano } & \multicolumn{8}{|c|}{ Resultados } \\
\hline & A & B & C & D & E & $\mathrm{F}$ & G & $\mathrm{H}$ & I & J & K & L & M & N & 0 & $P$ & Q \\
\hline & 4 & 6 & 2,5 & 10 & 9 & 12 & 7 & 9 & 16 & 5 & 1 & 2,5 & 8 & 13 & 11 & 14 & 15 \\
\hline$M$ & 6,18 & 5,90 & 6,22 & 5,12 & 5,51 & 4,94 & 5,87 & 5,19 & 4,21 & 6,10 & 6,27 & 6,22 & 5,54 & 4,58 & 5,06 & 4,56 & 4,23 \\
\hline Md & 7 & 6 & 7 & 6 & 6 & 5 & 6 & 6 & 4 & 6 & 7 & 7 & 6 & 5 & 6 & 5 & 5 \\
\hline $\mathrm{DP}$ & 1,17 & 1,28 & 1,07 & 1,86 & 1,57 & 1,84 & 1,27 & 1,85 & 2,21 & 1,15 & 1,10 & 0,97 & 1,59 & 2,07 & 1,85 & 2,12 & 2,39 \\
\hline$R$ & 6 & 6 & 6 & 6 & 6 & 6 & 6 & 6 & 6 & 6 & 6 & 4 & 6 & 6 & 6 & 6 & 6 \\
\hline Min - Max & $1-7$ & $1-7$ & $1-7$ & $1-7$ & $1-7$ & $1-7$ & $1-7$ & $1-7$ & $1-7$ & $1-7$ & $1-7$ & $3-7$ & $1-7$ & $1-7$ & $1-7$ & $1-7$ & $1-7$ \\
\hline$\%(5-7)^{*}$ & 93,6 & 89,5 & 94,3 & 74,2 & 80 & 68 & 86,4 & 72,8 & 58,4 & 92 & 93,6 & 92 & 80 & 61,6 & 71,8 & 61,3 & 69,6 \\
\hline
\end{tabular}

Nota. $M$ = Média; $M d$ = mediana; $D P$ = Desvio-padrão; $\mathrm{R}=$ Amplitude; * percentagem de respostas obtidas nos pontos 5,6 e 7 da escala de Likert de 7 pontos em que o ponto mínimo é 1 (discordo totalmente) e o ponto máximo é de 7 (concordo totalmente). Cores relativas às dimensões/áreas: Ato/agir $\rightarrow$ azul $=$ prazer, amarelo $=$ interesse, verde $=$ social; Capital Humano $\rightarrow$ amarelo $=$ material-egoísta, verde $=$ egoísta; Resultados $\rightarrow$ verde $=$ altruísta, amarelo $=$ necessidade e azul $=$ dever moral.

Tabela 3

Medidas de Tendência Central, de Dispersão e de Comparação de Grupos (U de Mann Whitney) na Motivação para o Voluntariado Consoante o Sexo

\begin{tabular}{|c|c|c|c|c|c|c|c|c|c|c|c|c|c|c|c|c|c|c|}
\hline & & \multicolumn{6}{|c|}{ Agir } & \multicolumn{4}{|c|}{ Capital Humano } & \multicolumn{7}{|c|}{ Resultados } \\
\hline & & A & B & C & D & $\mathrm{E}$ & $\mathrm{F}$ & G & $\mathrm{H}$ & I & $\mathrm{J}$ & $\mathrm{K}$ & L & M & $\mathrm{N}$ & 0 & $\mathrm{P}$ & Q \\
\hline \multicolumn{19}{|l|}{ Sexo } \\
\hline \multirow{4}{*}{ M } & $M$ & 6,22 & 5,85 & 6,28 & 5,02 & 5,45 & 4,98 & 5,98 & 5,47 & 4,17 & 6,19 & 6,37 & 6,42 & 5,83 & 4,69 & 5,26 & 4,73 & 4,35 \\
\hline & $M d$ & 7 & 6 & 7 & 6 & 6 & 5 & 6 & 6 & 4 & 7 & 7 & 7 & 6 & 5 & 6 & 5 & 5 \\
\hline & $D P$ & 1,20 & 1,40 & 1,05 & 1,96 & 1,70 & 1,93 & 1,22 & 1,71 & 2,29 & 1,08 & 1,03 & 0,74 & 1,49 & 2,14 & 1,90 & 2,13 & 2,35 \\
\hline & $R$ & 6 & 6 & 6 & 6 & 6 & 6 & 6 & 6 & 6 & 6 & 6 & 3 & 6 & 6 & 6 & 6 & 6 \\
\hline \multirow{4}{*}{$\mathrm{F}$} & $M$ & 6,10 & 6,00 & 6,08 & 5,32 & 5,61 & 4,83 & 5,63 & 4,66 & 4,37 & 5,88 & 6,05 & 5,85 & 5,00 & 4,37 & 4,66 & 4,24 & 4,07 \\
\hline & Md & 6 & 6 & 6,5 & 6 & 6 & 5 & 6 & 5 & 5 & 6 & 6 & 6 & 5 & 5 & 5 & 5 & 5 \\
\hline & $D P$ & 1,14 & 1,05 & 1,12 & 1,67 & 1,30 & 1,69 & 1,36 & 2,03 & 2,02 & 1,25 & 1,20 & 1,22 & 1,67 & 1,97 & 1,74 & 2,10 & 2,46 \\
\hline & $R$ & 4 & 4 & 4 & 6 & 5 & 6 & 5 & 6 & 6 & 5 & 4 & 6 & 6 & 6 & 6 & 6 & 6 \\
\hline M vs. F & & + & - & + & - & - & + & + & $+*$ & - & + & + & $+^{*}$ & $+^{*}$ & + & $+*$ & + & + \\
\hline
\end{tabular}

Nota. $\mathrm{M}=$ Masculino; $\mathrm{F}=$ Feminino. $M=$ Média; $M d=$ mediana; $\mathrm{DP}=$ Desvio-padrão; $\mathrm{R}=$ Amplitude. Cores relativas às dimensões/áreas: Ato/agir $\rightarrow$ azul $=$ prazer, amarelo = interesse, verde = social; Capital Humano $\rightarrow$ amarelo $=$ material-egoísta, verde $=$ egoísta; Resultados $\rightarrow$ verde $=$ altruísta, amarelo $=$ necessidade e azul $=$ dever moral 
Tabela 4

Medidas de Tendência Central, de Dispersão e de Comparação de Grupos (Kruskal-Wallis e U de Mann Whitney) na Motivação para o Voluntariado Consoante a Idade

\begin{tabular}{|c|c|c|c|c|c|c|c|c|c|c|c|c|c|c|c|c|c|c|}
\hline & & \multicolumn{6}{|c|}{ Agir } & \multicolumn{3}{|c|}{ Capital Humano } & \multicolumn{8}{|c|}{ Resultados } \\
\hline & & A & B & $C$ & $\mathrm{D}$ & $E$ & $\mathrm{~F}$ & G & $\mathrm{H}$ & I & $J$ & K & L & M & $\mathrm{N}$ & $\mathrm{O}$ & $\mathrm{P}$ & Q \\
\hline \multicolumn{19}{|c|}{ Idade } \\
\hline \multirow{4}{*}{ A } & $M$ & 6,33 & 6,01 & 6,35 & 5,10 & 5,69 & 4,75 & 5,70 & 5,00 & 3,91 & 6,13 & 6,33 & 6,19 & 5,44 & 4,34 & 4,94 & 4,38 & 3,91 \\
\hline & Md & 7 & 6 & 7 & 6 & 6 & 5 & 6 & 5 & 4 & 7 & 7 & 7 & 6 & 5 & 5 & 5 & 4 \\
\hline & $D P$ & 0,88 & 1,04 & 0,92 & 1,83 & 1,42 & 1,85 & 1,32 & 1,99 & 2,14 & 1,11 & 1,06 & 1,04 & 1,65 & 2,02 & 1,89 & 2,11 & 2,39 \\
\hline & $R$ & 4 & 4 & 4 & 6 & 6 & 6 & 5 & 6 & 6 & 6 & 5 & 4 & 6 & 6 & 6 & 6 & 6 \\
\hline \multirow{4}{*}{ B } & $M$ & 6,03 & 5,73 & 6,08 & 5,08 & 5,15 & 5,20 & 6,33 & 5,55 & 4,68 & 6,10 & 6,20 & 6,30 & 5,75 & 4,85 & 5,28 & 4,85 & 4,80 \\
\hline & Md & 7 & 6 & 7 & 6 & 6 & 6 & 7 & 6 & 6 & 7 & 7 & 7 & 6 & 6 & 6 & 6 & 6 \\
\hline & $D P$ & 1,56 & 1,68 & 1,25 & 2,02 & 1,83 & 1,88 & 0,86 & 1,58 & 2,36 & 1,26 & 1,22 & 0,85 & 1,56 & 2,20 & 1,88 & 2,11 & 2,34 \\
\hline & $R$ & 6 & 6 & 6 & 6 & 6 & 6 & 3 & 6 & 6 & 6 & 6 & 3 & 6 & 6 & 6 & 6 & 6 \\
\hline \multirow{4}{*}{$C$} & $M$ & 5,20 & 5,60 & 5,40 & 5,80 & 5,60 & 5,80 & 5,00 & 5,40 & 5,20 & 5,60 & 6,00 & 6,00 & 5,60 & 6,20 & 5,40 & 5,00 & 4,80 \\
\hline & Md & 6 & 6 & 6 & 6 & 6 & 6 & 6 & 6 & 5 & 6 & 6 & 6 & 6 & 6 & 5 & 6 & 6 \\
\hline & $D P$ & 1,30 & 1,14 & 1,34 & 1,10 & 1,52 & 0,84 & 2,24 & 0,89 & 1,30 & 0,89 & 0,00 & 0,71 & 0,55 & 0,45 & 0,55 & 2,35 & 2,17 \\
\hline & $R$ & 3 & 3 & 3 & 3 & 4 & 2 & 5 & 2 & 3 & 2 & 0 & 2 & 1 & 1 & 1 & 6 & 5 \\
\hline \multicolumn{2}{|c|}{$A$ vs. $B$} & + & + & + & + & + & - & $-*$ & - & + & - & + & - & - & - & - & - & - \\
\hline \multicolumn{2}{|c|}{ A vs. $C$} & + & + & - & - & - & - & + & - & + & + & + & + & - & $-*$ & + & + & - \\
\hline \multicolumn{2}{|c|}{ B vs. C } & + & + & + & - & - & - & + & + & + & + & + & + & + & - & - & - & $=$ \\
\hline
\end{tabular}

Nota. $\mathrm{A}$ = idades inferiores a 40 anos; $\mathrm{B}=$ idades compreendidas entre 40-59 anos; $\mathrm{C}=$ idades superiores a 60 anos. $M=M e ́ d i a ; M d=$ mediana; $D P=$ Desvio-padrão; $\mathrm{R}=\mathrm{Amplitude}$. Cores relativas às dimensões/áreas: Ato/agir $\rightarrow$ azul $=$ prazer, amarelo $=$ interesse, verde $=$ social; Capital Humano $\rightarrow$ amarelo $=$ material-egoísta, verde $=$ egoísta; Resultados $\rightarrow$ verde $=$ altruísta, amarelo $=$ necessidade e azul $=$ dever moral .

Na Tabela 5 constam as pontuações dos diferentes itens consoante a escolaridade. Existem diferenças em dois itens, o "A - Sinto-me satisfeito a fazer voluntariado" e o "H - Ao voluntariar-me melhoro a minha imagem para com a comunidade". A pontuação do item "A - Sinto-me satisfeito a fazer voluntariado", relativo ao indicador "prazer" do "Agir" apresenta, segundo o teste KruskalWallis, diferenças estatisticamente significativas, consoante o nível de escolaridade (Gp1, $n=37$ : básica; Gp2, $n=74$ : secundária; Gp3, $n=13: 10^{\circ} 2 .^{\circ}$ ciclo $), \chi^{2}(2, n=125)=7,72$, $p=0,021$. As diferenças, no caso do item "A - Sinto-me satisfeito a fazer voluntariado", são constatadas nas pontuações entre o Gp1 (básico) e o Gp2 (secundário) (Md $=6$ vs. $M d=7), U=964,00 ; z=-2,781 ; p=0,005$. Relativamente ao item " $\mathrm{H}$ - Ao voluntariar-me melhoro a minha imagem para com a comunidade", indicador "egoísta" do "capital humano", este apresenta igualmente diferenças estatisticamente significativas consoante o nível de escolaridade (Gp1, $n=37$ : básica; Gp2, $n=74:$ secundária; Gp3, $n=13: 10^{\circ} 2 .^{\circ}$ ciclo $), \chi 2(2, n=125)=$ 6,39; $p=0,041$. As diferenças, no caso do item " $\mathrm{H}$ - Ao voluntariar-me melhoro a minha imagem para com a comunidade", são constatadas nas pontuações entre o Gp1 (básico) e o Gp3 $\left(1 .^{\circ}\right.$ e $2 .^{\circ}$ ciclo) ( $M d=6$ vs. $\left.M d=5\right), U=$ 138,00; $z=-2,339, p=0,019$.

A Tabela 6 apresenta as pontuações dos diferentes itens consoante o rendimento. Segundo o teste KruskalWallis existem diferenças estatisticamente significativas, nas pontuações dos itens "J - Estou a fazer algo útil”, “K É uma grande oportunidade para ajudar quem mais precisa na comunidade" e " $M$ - Voluntario-me porque estou a contribuir para o futuro da empresa/organização", relativas ao indicador "egoísta" dos "resultados" consoante o rendimento (Gp1, $n=82$ : 500-1000; Gp2, $n=$ 15: 1000-1500, Gp3, $n=4:(500-2500) \chi^{2}(2, n=126)=6,272 ; p=$ $0,043, \chi^{2}(2, n=126)=9,750 ; p=0,008$ e $\chi^{2}(2, n=126)=8,808 ; p=$ 0,012 , respetivamente. As diferenças, no caso do item " J Estou a fazer algo útil”, são constatadas nas pontuações entre o Gp1 (500-1000) e o Gp3 (1500-2500) (Md= 7 vs. Md = 5), $U=59,00 ; z=-2,328, p=0,020$. No item “K - É uma grande oportunidade para ajudar quem mais precisa na comunidade", são constatadas diferenças nas pontuações tanto entre o Gp1 (500-1000) e o Gp3 (1500-2500) ( $M d=7$ vs. $M d=4), U=27,00 ; z=-3,133, p=0,002$, como entre $o$ Gp2 (1000-1500) e o Gp3 (1500-2500) (Md= 7 vs. $M d=4), U$ $=8,00 ; z=-2,310 ; p=0,021$. No que concerne ao item " $M-$ Voluntario-me porque estou a contribuir para o futuro da empresa/organização", são constatadas diferenças nas pontuações entre o Gp1 (500-1000) e o Gp3 (1500-2500) $(M d=6$ vs. $M d=4), U=30,50 ; z=-2,858 ; p=0,004$ como entre o Gp2 (1000-1500) e o Gp3 (1500-2500) (Md = 6 vs. $M d=4), U=8,50 ; z=-2,243 ; p=0,025$.

A Tabela 7 apresenta as pontuações dos diferentes itens consoante os anos de voluntariado. Foram observadas diferenças estatisticamente significativas em itens dos "resultados", nomeadamente no indicador “ $\mathrm{K}$ É uma grande oportunidade para ajudar quem mais precisa na comunidade", indicador "altruísta" e "O Voluntario-me porque a empresa/organização beneficia com a minha experiência", indicador "necessidade", quando comparamos os bombeiros com diferentes anos de voluntariado (Gp1, $n=12:>2$ anos; Gp2, $n=48: 2-10$ 
anos, Gp3, $n=66$ : >10 anos), $\chi^{2}(2, n=126)=7,173 ; p=0,028$, e $\chi^{2}(2, n=126)=9,160 ; p=0,010$, respetivamente. No que concerne ao indicador "K - É uma grande oportunidade para ajudar quem mais precisa na comunidade", indicador "altruísta", são constatadas diferenças tanto nas pontuações entre o Gp1 (> 2 anos) e o Gp2 (2-10 anos) (Md $=6$ vs. $M d=7), U=162,50 ; z=-2,496 ; p=0,013$, como entre o Gp1 (> 2 anos) e o Gp3 (> 10 anos) ( $M d=6$ vs. $M d=$ 7), $U=231,50 ; z=-2,504 ; p=0,012$. Relativamente ao indicador "O Voluntario-me porque a empresa/ organização beneficia com a minha experiência", indicador "necessidade", são constatadas diferenças nas pontuações entre o Gp2 (2-10 anos) e o Gp3 (> 10 anos) $(M d=5$ vs. $M d=6), U=1032,00 ; z=-2,95 ; p=0,003$.

\section{Tabela 5}

Medidas de Tendência Central, de Dispersão e de Comparação de Grupos (Kruskal-Wallis e U de Mann Whitney) na Motivação para o Voluntariado Consoante o Nível de Escolaridade

\begin{tabular}{|c|c|c|c|c|c|c|c|c|c|c|c|c|c|c|c|c|c|c|}
\hline & & \multicolumn{6}{|c|}{ Agir } & \multicolumn{3}{|c|}{ Capital Humano } & \multicolumn{8}{|c|}{ Resultados } \\
\hline \multirow{2}{*}{\multicolumn{2}{|c|}{$\begin{array}{l}\text { Escola- } \\
\text { ridade }\end{array}$}} & A & B & $C$ & $\mathrm{D}$ & $E$ & $\mathrm{~F}$ & $G$ & $\mathrm{H}$ & I & J & $\mathrm{K}$ & L & $M$ & $\mathrm{~N}$ & $\mathrm{O}$ & $\mathrm{P}$ & Q \\
\hline & & & & & & & & & & & & & & & & & & \\
\hline \multirow{4}{*}{ A } & $M$ & 5,73 & 5,57 & 6,05 & 5,27 & 5,35 & 5,16 & 6,05 & 5,76 & 4,51 & 6,00 & 6,27 & 6,41 & 5,89 & 4,86 & 5,41 & 4,78 & 4,81 \\
\hline & Md & 6 & 6 & 6 & 6 & 6 & 5 & 6 & 6 & 5 & 6 & 7 & 7 & 6 & 5 & 6 & 6 & 6 \\
\hline & $D P$ & 1,54 & 1,69 & 1,35 & 1,84 & 1,74 & 1,74 & 1,20 & 1,52 & 2,13 & 1,22 & 1,12 & 0,76 & 1,24 & 2,08 & 1,71 & 2,11 & 2,32 \\
\hline & $R$ & 6 & 6 & 6 & 6 & 6 & 6 & 6 & 6 & 6 & 6 & 6 & 3 & 6 & 6 & 6 & 6 & 6 \\
\hline \multirow{4}{*}{$B$} & $M$ & 6,41 & 6,07 & 6,32 & 5,03 & 5,70 & 4,85 & 5,91 & 5,07 & 4,01 & 6,26 & 6,39 & 6,23 & 5,51 & 4,32 & 4,88 & 4,47 & 3,88 \\
\hline & Md & 7 & 6 & 7 & 6 & 6 & 5 & 6 & 5 & 4 & 7 & 7 & 7 & 6 & 5 & 6 & 5 & 4 \\
\hline & $D P$ & 0,94 & 1,07 & 0,92 & 1,95 & 1,33 & 1,89 & 1,14 & 1,90 & 2,17 & 0,94 & 0,86 & 0,96 & 1,64 & 2,13 & 1,97 & 2,12 & 2,38 \\
\hline & $R$ & 4 & 5 & 3 & 6 & 6 & 6 & 4 & 6 & 6 & 3 & 3 & 3 & 6 & 6 & 6 & 6 & 6 \\
\hline \multirow{4}{*}{$C$} & $M$ & 6,31 & 6,00 & 6,15 & 5,15 & 4,77 & 4,62 & 5,15 & 4,31 & 4,38 & 5,46 & 5,62 & 5,69 & 4,77 & 5,08 & 5,15 & 4,31 & 4,54 \\
\hline & Md & 7 & 6 & 7 & 5 & 5 & 5 & 6 & 5 & 6 & 6 & 7 & 6 & 4 & 6 & 5 & 5 & 6 \\
\hline & $D P$ & 0,85 & 0,91 & 0,99 & 1,52 & 2,17 & 1,85 & 1,95 & 2,10 & 2,75 & 1,76 & 1,89 & 1,38 & 2,05 & 1,61 & 1,57 & 2,29 & 2,54 \\
\hline & $R$ & 2 & 3 & 2 & 6 & 6 & 5 & 5 & 6 & 6 & 6 & 5 & 4 & 6 & 5 & 5 & 6 & 6 \\
\hline \multicolumn{2}{|c|}{ A vs. B } & $-^{*}$ & - & - & + & - & + & + & + & + & - & - & + & + & + & + & + & + \\
\hline \multicolumn{2}{|c|}{ A vs. $C$} & - & - & - & + & + & + & + & $+^{*}$ & + & + & + & + & + & - & + & + & + \\
\hline \multicolumn{2}{|c|}{ B vs. C } & + & + & + & - & + & + & + & + & - & + & + & + & + & - & - & + & - \\
\hline
\end{tabular}

Nota. $\mathrm{A}=$ escolaridade básica; $\mathrm{B}=$ secundária; $\mathrm{C}=1 .^{\circ}$ e $2 .^{\circ}$ ciclo. $M=$ Média; $M d=$ mediana; $D P=$ Desvio-padrão; $\mathrm{R}=$ Amplitude. Cores relativas às dimensões/áreas: Ato/agir $\rightarrow$ azul $=$ prazer amarelo $=$ interesse, verde $=$ social; Capital Humano $\rightarrow$ amarelo $=$ material-egoísta, verde $=$ egoísta; Resultados $\rightarrow$ verde $=$ altruísta, amarelo $=$ necessidade e azul $=$ dever moral

\section{Tabela 6}

Medidas de Tendência Central, de Dispersão e de Comparação de Grupos (Kruskal-Wallis e U de Mann Whitney) da Motivação para o Voluntariado Consoante o Rendimento

\begin{tabular}{|c|c|c|c|c|c|c|c|c|c|c|c|c|c|c|c|c|c|c|}
\hline \multirow{3}{*}{\multicolumn{2}{|c|}{$\begin{array}{l}\text { Rendi- } \\
\text { mento }\end{array}$}} & \multicolumn{6}{|c|}{ Agir } & \multicolumn{3}{|c|}{ Capital Humano } & \multicolumn{8}{|c|}{ Resultados } \\
\hline & & \multirow[t]{2}{*}{ A } & \multirow[t]{2}{*}{ B } & \multirow[t]{2}{*}{ C } & \multirow[t]{2}{*}{ D } & \multirow[t]{2}{*}{$\mathrm{E}$} & \multirow[t]{2}{*}{$\mathrm{F}$} & \multirow[t]{2}{*}{ G } & \multirow[t]{2}{*}{$\mathrm{H}$} & \multirow[t]{2}{*}{1} & J & \multirow[t]{2}{*}{ K } & \multirow[t]{2}{*}{ L } & \multirow[t]{2}{*}{ M } & \multirow[t]{2}{*}{$\mathrm{N}$} & \multirow[t]{2}{*}{0} & \multirow[t]{2}{*}{$P$} & \multirow[t]{2}{*}{ Q } \\
\hline & & & & & & & & & & & & & & & & & & \\
\hline \multirow{4}{*}{ A } & M & 6,18 & 5,86 & 6,14 & 5,20 & 5,62 & 5,09 & 5,94 & 5,46 & 4,38 & 6,17 & 6,37 & 6,22 & 5,80 & 4,60 & 5,25 & 4,52 & 4,29 \\
\hline & Md & 7,0 & 6,0 & 6,0 & 6,0 & 6,0 & 5,0 & 6,0 & 6,0 & 5,0 & 7,0 & 7,0 & 6,5 & 6,0 & 5,0 & 6,0 & 5,0 & 5,0 \\
\hline & $D P$ & 1,17 & 1,31 & 1,12 & 1,79 & 1,51 & 1,75 & 1,21 & 1,69 & 2,15 & 1,12 & 1,00 & 0,94 & 1,40 & 2,03 & 1,75 & 2,03 & 2,42 \\
\hline & $R$ & 6 & 6 & 6 & 6 & 6 & 6 & 6 & 6 & 6 & 6 & 6 & 3 & 6 & 6 & 6 & 6 & 6 \\
\hline \multirow{4}{*}{ B } & M & 6,13 & 6,07 & 6,07 & 5,13 & 5,13 & 4,80 & 5,60 & 4,87 & 4,73 & 5,80 & 6,13 & 6,00 & 5,33 & 5,20 & 4,93 & 5,07 & 5,27 \\
\hline & Md & 6 & 6 & 6 & 6 & 6 & 5 & 6 & 5 & 6 & 6 & 7 & 6 & 6 & 6 & 5 & 6 & 6 \\
\hline & $D P$ & 1,13 & 1,10 & 1,16 & 1,96 & 1,77 & 2,01 & 1,64 & 2,13 & 2,46 & 1,52 & 1,36 & 1,25 & 1,68 & 2,08 & 1,83 & 2,31 & 2,19 \\
\hline & $R$ & 4 & 4 & 4 & 6 & 6 & 6 & 5 & 6 & 6 & 6 & 5 & 4 & 5 & 6 & 6 & 6 & 6 \\
\hline \multirow{4}{*}{ C } & $M$ & 5,00 & 4,80 & 5,60 & 4,40 & 4,00 & 4,00 & 5,60 & 5,40 & 4,40 & 5,40 & 4,60 & 5,80 & 3,80 & 4,20 & 5,40 & 4,80 & 5,00 \\
\hline & Md & 5 & 5 & 5 & 5 & 6 & 5 & 6 & 6 & 4 & 5 & 4 & 6 & 4 & 4 & 6 & 5 & 6 \\
\hline & $D P$ & 2,45 & 2,28 & 0,89 & 2,07 & 2,74 & 2,35 & 2,07 & 2,07 & 2,61 & 1,14 & 1,82 & 1,30 & 2,39 & 2,39 & 1,82 & 2,49 & 2,55 \\
\hline & $R$ & 6 & 6 & 2 & 5 & 5 & 5 & 5 & 5 & 6 & 3 & 4 & 3 & 6 & 6 & 4 & 6 & 6 \\
\hline \multicolumn{2}{|c|}{ A vs. B } & + & - & + & + & + & + & + & + & - & + & + & + & - & - & + & - & - \\
\hline \multicolumn{2}{|c|}{ A vs. C } & + & + & + & + & + & + & + & + & - & $+*$ & -* & + & $+^{*}$ & + & - & - & - \\
\hline \multicolumn{2}{|c|}{ B vs. C } & + & + & + & + & + & + & $=$ & - & + & + & $+^{*}$ & + & $+^{*}$ & + & - & - & - \\
\hline
\end{tabular}

Notas: $\mathrm{A}=$ rendimento inferior a 500€; $\mathrm{B}=$ rendimento entre 1000 e $1500 € ; \mathrm{C}=$ rendimento entre 1500-2500. $M=$ Média; $M d=$ mediana; $D P=$ Desvio-padrão; $\mathrm{R}=\mathrm{Amplitude}$. Cores relativas às dimensões/áreas: Ato/agir $\rightarrow$ azul $=$ prazer, amarelo $=$ interesse, verde $=$ social; Capital Humano $\rightarrow$ amarelo $=$ material-egoísta, verde $=$ egoísta; Resultados $\rightarrow$ verde $=$ altruísta, amarelo = necessidade e azul = dever moral. 
Tabela 7

Medidas de Tendência Central, de Dispersão e de Comparação de Grupos (Kruskal-Wallis e U de Mann Whitney) na Motivação para o Voluntariado Consoante os Anos de Voluntariado

\begin{tabular}{|c|c|c|c|c|c|c|c|c|c|c|c|c|c|c|c|c|c|c|}
\hline & & \multicolumn{6}{|c|}{ Agir } & \multicolumn{3}{|c|}{ Capital Humano } & \multicolumn{8}{|c|}{ Resultados } \\
\hline & & $A$ & B & C & $\mathrm{D}$ & $E$ & $\mathrm{~F}$ & G & $\mathrm{H}$ & 1 & J & K & $\mathrm{L}$ & $M$ & $\mathrm{~N}$ & 0 & $P$ & Q \\
\hline \multicolumn{19}{|c|}{ Anos } \\
\hline \multirow{4}{*}{ A } & $M$ & 5,67 & 5,33 & 5,92 & 5,25 & 4,92 & 5,00 & 5,33 & 4,75 & 5,25 & 5,58 & 5,50 & 5,83 & 5,00 & 4,75 & 5,25 & 4,75 & 4,67 \\
\hline & Md & 6,00 & 5,50 & 6,00 & 5,50 & 5,00 & 5,00 & 5,50 & 5,00 & 5,50 & 6,00 & 6,00 & 6,00 & 5,00 & 5,00 & 5,00 & 4,50 & 4,50 \\
\hline & $D P$ & 1,23 & 1,23 & 1,31 & 1,14 & 1,51 & 1,41 & 1,56 & 1,14 & 1,29 & 1,00 & 1,31 & 1,03 & 1,71 & 1,66 & 1,14 & 1,29 & 1,83 \\
\hline & $R$ & 4 & 4 & 4 & 4 & 6 & 5 & 5 & 4 & 4 & 3 & 4 & 3 & 5 & 6 & 3 & 4 & 5 \\
\hline \multirow{4}{*}{ B } & M & 6,17 & 6,09 & 6,24 & 4,87 & 5,62 & 4,74 & 5,79 & 5,00 & 4,34 & 6,23 & 6,38 & 6,17 & 5,38 & 4,28 & 4,48 & 4,54 & 3,68 \\
\hline & Md & 6 & 6 & 7 & 6 & 6 & 5 & 6 & 5 & 5 & 7 & 7 & 7 & 6 & 5 & 5 & 5 & 4 \\
\hline & $D P$ & 1,03 & 0,98 & 0,95 & 2,00 & 1,17 & 1,88 & 1,32 & 2,03 & 2,33 & 0,91 & 0,97 & 1,05 & 1,70 & 2,02 & 1,97 & 2,19 & 2,42 \\
\hline & $R$ & 4 & 3 & 3 & 6 & 4 & 6 & 5 & 6 & 6 & 3 & 4 & 3 & 6 & 6 & 6 & 6 & 6 \\
\hline \multirow{4}{*}{ C } & $M$ & 6,29 & 5,88 & 6,26 & 5,28 & 5,55 & 5,06 & 6,03 & 5,41 & 3,92 & 6,09 & 6,33 & 6,32 & 5,76 & 4,76 & 5,44 & 4,53 & 4,55 \\
\hline & $M d$ & 7,00 & 6,00 & 7,00 & 6,00 & 6,00 & 6,00 & 6,00 & 6,00 & 4,00 & 6,50 & 7,00 & 7,00 & 6,00 & 5,00 & 6,00 & 5,00 & 5,50 \\
\hline & $\mathrm{DP}$ & 1,25 & 1,45 & 1,11 & 1,87 & 1,82 & 1,89 & 1,16 & 1,81 & 2,21 & 1,30 & 1,10 & 0,90 & 1,48 & 2,18 & 1,78 & 2,21 & 2,41 \\
\hline & $R$ & 6 & 6 & 6 & 6 & 6 & 6 & 6 & 6 & 6 & 6 & 6 & 4 & 6 & 6 & 6 & 6 & 6 \\
\hline \multicolumn{2}{|c|}{ A vs. B } & - & - & - & + & - & + & - & - & + & - & -* & - & - & + & + & + & + \\
\hline \multicolumn{2}{|c|}{ A vs. C } & - & - & - & - & + & - & - & - & + & - & $-*$ & - & - & - & - & + & + \\
\hline \multicolumn{2}{|c|}{ B vs. C } & - & + & - & - & + & - & - & - & + & + & + & - & - & - & $-*$ & + & - \\
\hline
\end{tabular}

Notas: $\mathrm{A}=$ inferior a 2 anos de voluntariado; $\mathrm{B}=$ entre 2 e 10 anos de voluntariado $; \mathrm{C}=$ superior a 10 anos de voluntariado. $M=\mathrm{Mé}$ dia; $M d=$ mediana; $\mathrm{DP}=\mathrm{Desvio}$-padrão; $\mathrm{R}=$ Amplitude. Cores relativas às dimensões/áreas: Ato/agir $\rightarrow$ azul $=$ prazer, amarelo $=$ interesse, verde $=$ social; Capital Humano $\rightarrow$ amarelo $=$ material-egoísta, verde $=$ egoísta; Resultados $\rightarrow$ verde $=$ altruísta, amarelo $=$ necessidade e azul $=$ dever moral.

\section{DISCUSSÃO E CONCLUSÃO}

As Associações de Bombeiros Humanitários desempenham um papel importante, principalmente em países como Portugal, onde o número de incêndios é elevado, dando origem a consequências económicas, sociais, materiais e humanas de vária ordem, designadamente destruição dos recursos naturais, custos elevados na afetação de meios materiais e humanos, destruição de bens materiais e, inclusivamente, perda de vidas humanas. O recrutamento e a retenção de voluntários nas Associações de Bombeiros Humanitários tornam-se, assim, assuntos de particular relevância que importa analisar. O nosso estudo tenta preencher um défice que existe na literatura sobre este assunto, pois são muito escassas as investigações sobre os fatores que determinam a motivação dos bombeiros voluntários, quer a nível internacional, quer em Portugal.

Os resultados globais do nosso estudo são consistentes com investigações precedentes sobre os fatores que explicam a motivação para o voluntariado. $\mathrm{Na}$ verdade, o altruísmo é o fator dominante na explicação do comportamento dos bombeiros voluntários, uma vez que entre as cinco razões mais pontuadas para o voluntariado, três estão diretamente ligadas a indicadores de altruísmo.
De acordo com a tabela 2, o item de valor mais elevado é o $\mathrm{K}$ : Eu voluntario-me porque "É uma grande oportunidade para ajudar quem mais precisa” logo seguido do L "Estou a contribuir para uma sociedade melhor". Refira-se ainda a pontuação alta do item J "Estou a fazer algo útil". O número de vezes que os diversos itens são pontuados entre 5 a 7 na escala de Likert, valor que é fornecido pela última linha da tabela 2, revela igualmente a importância elevada das razões altruístas. Também de acordo com a literatura segue-se, depois da motivação pelo altruísmo, aquela que resulta do próprio ato de voluntariado, tal como é ilustrado pelos valores do item C: "Fico feliz com as ações de voluntariado" (o segundo item mais pontuado, juntamente com o L, atrás referido) e do item A: "Sinto-me satisfeito a fazer voluntariado", o quarto item mais valorizado. Estes resultados são consistentes com os estudos de Ward e Mckillop (2011) e Ziemek (2006).

As razões consideradas menos importantes, que fundamentam a motivação dos bombeiros voluntários, estão ligadas à categoria “capital humano", em particular à questão I (Voluntario-me porque estou a ganhar experiência prática para um emprego pago no futuro) e à categoria "resultados" na questão Q, relacionada com o dever moral, o que mais uma vez está de acordo com as investigações realizadas por outros autores (cf. Ward e Mckillop, 2011). 
Todavia, quando desagregamos a análise através das características dos voluntários (sexo, idade, grau de escolarização, rendimento e anos de voluntariado), os resultados nem sempre coincidem com aqueles que se encontram na literatura especializada.

Em relação ao sexo, como atrás se referiu, encontram-se diferenças estatisticamente significativas em alguns indicadores ligados ao altruísmo (itens $L$ e $M$ ), ao capital humano (item $\mathrm{H}$ ) e ao dever moral (item $\mathrm{O}$ ). De acordo com os nossos resultados, os homens são mais altruístas do que as mulheres, valorizam mais o capital humano e o dever moral. Desta forma, as diferenças nos resultados obtidos, quando confrontados com outras investigações, residem essencialmente no facto da motivação para o altruísmo encontrar valores mais elevados no sexo masculino. Refira-se, no entanto, que esta aparente diferença pode ter várias explicações. Em primeiro lugar, no caso dos bombeiros voluntários, estamos perante uma atividade muito particular que envolve uma situação de risco e que não tem sido considerada nos estudos sobre a motivação para o voluntariado. Esta situação de risco poderá ser mais facilmente assumida pelo sexo masculino, devido às condicionantes culturais próprias das sociedades ocidentais que remetem para a figura do homem enquanto "bravo", "corajoso", "capaz de tudo" (Honório, 2011, p. 10). Em segundo lugar, alguns estudos sobre o voluntariado têm chamado a atenção para o facto de a motivação segundo o sexo depender do tipo de voluntariado (Andreoni e Vesterlund, 2001) e do contexto cultural (Gong, Yan e Yang, 2014; Gneezy, Leonard e List, 2009). Associado a estes argumentos, convém também salientar que alguns estudos sobre motivação e género têm apresentado resultados ambíguos. Em suma, a afirmação generalizada de que as mulheres são mais altruístas parece, assim, um pouco precipitada, já que o altruísmo parece depender do contexto institucional e do tipo de atividade considerado.

O nosso estudo evidencia também que, em relação à variável "idade", as diferenças estatisticamente significativas encontram-se nos itens $\mathrm{G}$ e N, associados ao "capital humano" e à "necessidade". De acordo com os nossos resultados, a motivação pela melhoria do capital humano no escalão etário abaixo dos 40 anos tem uma importância mais baixa do que na classe situada entre os 40 e 59 anos, o que contraria os resultados de grande parte das investigações sobre o tema (ver, por exemplo, Peterson, 2004; Ziemek, 2006). Convém, no entanto, lembrar que a relação entre a idade e as razões sociais que motivam o voluntariado nem sempre é linear, pois alguns estudos mostram que os voluntários de maior idade podem também ser motivados pelo interesse próprio (Okun e Schuktz, 2003).

Em relação ao grau de escolarização, as diferenças significativas que os nossos resultados revelam situam-se no item $\mathrm{H}$, relacionado com o capital humano e $\mathrm{A}$, associado à satisfação obtida com as interações sociais estabelecidas através das atividades de voluntariado. A motivação pela necessidade de elevar o nível de capital humano é significativamente mais elevada nos voluntários com grau de escolarização básica, em relação aos que têm a escolarização secundária, o que está de acordo com os principais estudos atrás referidos (Clary et al.1996). Quanto ao item A, associado à satisfação obtida através do voluntariado, os nossos resultados mostram que as pontuações mais elevadas são atribuídas pelos indivíduos com grau secundário de escolarização, relativamente àqueles com grau de escolarização básico. Este resultado, embora não consistente com alguns estudos (Ward e Mckilop, 2011), pode ser explicado pelo facto do primeiro grupo centrar as suas principais motivações nas razões ligadas à melhoria do seu nível de capital humano e considerar menos importante o estabelecimento de interações sociais.

No nosso estudo, os níveis mais elevados dos escalões de rendimento não correspondem a valores mais altos nos indicadores altruístas (Dettoleneare et al., 2017), o que contraria alguns estudos sobre o tema, apesar de estes serem escassos, como atrás se referiu. Na verdade, as diferenças estatisticamente significativas apontam no sentido inverso, o que significa que níveis mais baixos de rendimento estão associados a valores mais elevados nas razões altruístas, refletidos nos itens $\mathrm{J}$, K e $\mathrm{M}$. Como foi salientado, outros estudos mostram que as relações entre aquelas variáveis não são lineares (Hackl et al., 2007). Assim, parece prematuro avançar no estabelecimento de relações definitivas entre rendimento e motivação, dada a escassez dos estudos e a ambiguidade dos resultados.

Quanto à relação entre níveis de rendimento e motivação, os nossos resultados são consistentes com os trabalhos de outros autores, onde se refere que os motivos que justificam a oferta de trabalho voluntário evoluem ao longo do tempo (Thomas e Finch, 1990; Tschirhart et al., 2001; Ward e Mckilop, 2011). As razões de motivação quando associadas aos anos de voluntariado mostram de forma clara que, quando o tempo de voluntariado na organização aumenta, os indicadores altruístas são mais elevados. Dito de outra forma, os voluntários experientes são mais altruístas do que os iniciantes.

Em suma, podemos retirar do nosso estudo algumas conclusões que terão utilidade no recrutamento e retenção dos bombeiros voluntários. Cabem, no entanto, algumas observações finais. Em primeiro lugar, o estudo sobre os fatores determinantes da motivação deve ser enquadrado no contexto sociocultural onde decorrem as atividades de voluntariado. Como já foi mostrado em outros estudos, a motivação para o voluntariado varia de país para país (Ziemek, 2006). Talvez por isso mesmo, os resultados das investigações realizadas sobre o tema são, por vezes, ambíguos. Daí a necessidade de se desenvolverem mais trabalhos comparativos a nível internacional. Por outro lado, o estudo da motivação no 
caso dos bombeiros voluntários não pode, também, ser desligado das especificidades da própria atividade, o que traz a necessidade de se aprofundarem os estudos sobre a motivação nas atividades que envolvem um risco para a segurança e para a integridade física dos participantes.

Conflito de interesses | Conflict of interest: nenhum | none.

Fontes de financiamento | Funding sources: nenhuma | none.

\section{Agradecimentos | acknowledgements}

Os autores agradecem à Ana Figueiredo e à Jéssica Pereira pelo seu trabalho na recolha de dados, sem o qual este estudo não teria sido possível.

\section{REFERÊNCIAS}

Anderson, K. (2003). Student volunteers: why hospitals must invest in their futures. International Journal of Health Care Quality Assurance, 16(2), vi-xiii. doi:10.1108/13660750310474517

Andreoni J. e Vesterlund L. (2001). Which is the fair sex? Gender differences in altruism. Quarterly Journal of Economics, 116, 293-312. doi:10.1162/003355301556419

Clary, E., Snyder, M. e Stukas, A. (1996). Volunteers' motivations: Findings from a national survey. Nonprofit and Voluntary Sector Quarterly, 25(4), 485-505. doi:10.1177/0899764096254006

Dettolenaere, J., Baert, S. e Willems, S. (2017). Volunteering, income and health. PLOS ONE, 12(3), Recuperado de http://journals.plos.org/ plosone/article?id=10.1371/journal.pone.0173139

Eckel C. e Grossman P. J. (1998). Are women less selfish than men? Evidence from dictator experiments, Economic Journal, 108, 726-735.

Gneezy, U., Leonard, K., e List, J. (2009). Gender differences in competition: evidence from a matrilineal society. Econometrica, 77(5), 1637-1664. doi: 10.3982/ECTA6690

Gong, B., Yan, H. e Yang, C. (2015). Gender differences in the dictator experiment: evidence from the matrilineal Mosuo and the patriarchal Yi. Experimental Economics, 18(2), 302-3013. doi: 10.1007/ s10683-014-9403-2.
Hackl, F., Halla, M. e Pruckner, G. J. (2007). Volunteering and income - the fallacy of the Good Samaritan? Kyklos, 60(1), 77-104. doi:10.1111/j. 1467-6435.2007.00360.x

Honório, M. D. (2011). Cabra-macho, sim senhor! Um estudo sobre a masculinidade no Nordeste do Brasil. Anais do XV Congresso Brasileiro de Sociologia, Curitiba, 26 a 29 de julho.

INE (2017). Portal das Estatísticas, Instituto Nacional de Estatística. Recuperado em www.ine.pt

Jager U. P., Kreutzer, K. e Beyes, T. (2009). Balancing acts: NPOleadership and volunteering. Financial Accountability and Management, 25(1), 79-97. doi:10.1111/j.1468-0408.2008.00466.x

Maclaran, P. e Caterrall, M. (2000). Bridging the knowledge divide. Journal of Marketing Management, 15, 635-646. doi: 10.1362/026725700785045958

Nichols, J. e Ralston, R. (2016). Talking 'bout my generational differences in the attitudes of volunteers at the 2012 Olympic Games. Voluntary Sector Review, 7(2), 127-147. doi:10.1332/204080516X14650415652348

Okun, M. e Scultz, M. (2003), Age and Motives for Volunteering: Testing Hypotheses Derived from Socioemotional Selectivity Theory. Psychology and Aging, 18(2), 231-239.

Peterson, D. (2004). Recruitment strategies for encouraging participation in corporate volunteer programs. Journal of Business Ethics, 49, 3713-82.

Themudo, N. (2009). Gender and the nonprofit sector. Nonprofit and Voluntary Sector Quarterly, 38(4), 663-683. doi: 10.1177/0899764009333957

Thomas, A. e Finch, H. (1990). On volunteering: a qualitative research study on images, motivations and experience. Voluntary Action Research Centre UK.

Tidwell, M. (2005). A social identity model of prosocial behaviours within non-profit organisations. Nonprofit Management and Leadership, 15, 449-450. doi:10.1002/nml.82

Tschirhart, M. Mesch, D., Miller, T. K. e Lee, G. (2001). Stipended volunteers: their goals, experiences, satisfaction, and likelihood of future service. Nonprofit and Voluntary Sector Quarterly, 30(3), 422-443. doi:10.1177/0899764001303002

Wilson J. (2000). Volunteering. Annual Review of Sociology, 26, 15-40.

Ward, A. M. e Mckillop, D. G (2011). An Examination of Volunteer Motivation in Credit Unions: Informing Volunteer Resource Management. Annals of Public and Cooperative Economics, 82(3), 253-275. doi:10.1111/j.1467-8292.2011.00438.x

Ziemek S. (2006). Economic analysis of volunteer's motivations - a crosscountry study. The Journal of Socio-Economics, 35, 532-555. doi: 10.1016/j.socec.2005.11.064 